\title{
Review of "Open Science"
}

\author{
Leonardo Candela ${ }^{1}$ \\ 1 Italian National Res earch Council
}

TYhis definition focus on a process (transition) that is not meant to be the primary target. Moreover, "new" is problematic because of timing ... how long is expected to be Open Science something new? 